\title{
Charge-Induced Structural Changes in a Single Molecule Investigated by Atomic Force Microscopy
}

\author{
Philipp Scheuerer, ${ }^{1, *}$ Laerte L. Patera, ${ }^{1}$ Felix Simbürger, ${ }^{1}$ Fabian Queck, ${ }^{1}$ Ingmar Swart, ${ }^{1,2}$ \\ Bruno Schuler, ${ }^{3,4}$ Leo Gross, ${ }^{3}$ Nikolaj Moll, ${ }^{3, \dagger}$ and Jascha Repp ${ }^{1}$ \\ ${ }^{1}$ Institute of Experimental and Applied Physics, University of Regensburg, 93053 Regensburg, Germany \\ ${ }^{2}$ Debye Institute for Nanomaterials Science, Utrecht University, PO Box 80 000, 3508 TA Utrecht, Netherlands \\ ${ }^{3}$ IBM Research-Zurich, 8803 Rüschlikon, Switzerland \\ ${ }^{4}$ The Molecular Foundry, Lawrence Berkeley National Laboratory, Berkeley, California 94720, USA
}

(Received 4 March 2019; revised manuscript received 12 June 2019; published 7 August 2019)

\begin{abstract}
Intramolecular structural relaxations occurring upon electron transfer are crucial in determining the rate of redox reactions. Here, we demonstrate that subangstrom structural changes occurring upon singleelectron charging can be quantified by means of atomically resolved atomic force microscopy (AFM) for the case of single copper(II)phthalocyanine ( $\mathrm{CuPc}$ ) molecules deposited on an ultrathin $\mathrm{NaCl}$ film. Imaging the molecule in distinct charge states (neutral and anionic) reveals characteristic differences in the AFM contrast. In comparison to density functional theory simulations these changes in contrast can be directly related to relaxations of the molecule's geometric structure upon charging. The dominant contribution arises from a nonhomogeneous vertical relaxation of the molecule, caused by a change in the electrostatic interaction with the surface.
\end{abstract}

DOI: 10.1103/PhysRevLett.123.066001

Electron transfer lies at the heart of many chemical and biological processes [1-5]. Changes in the redox state originating from electron transfer lead to structural relaxations of the molecule and the environment (e.g., solvation shells), which, in turn act back on the electronic states. The resulting energy gain, the Marcus reorganization energy [2], governs the rate of redox reactions, being decisive also for hopping conduction in organic materials [6-8]. Scanning probe microscopy has provided insight into the mechanisms governing the electron transfer at the single-molecule level [9-26]. For example, the influence of an additional charge on the tunneling barrier was revealed by means of scanning tunneling microscopy (STM) [13]. Recent experiments based on atomic force microscopy (AFM) for detecting single-electron tunneling events in individual molecules deposited on non conductive substrates allowed determining the reorganization energy [25] and imaging orbital changes upon electron transfer with angstrom resolution [26]. Whereas all these effects are governed by the complex interplay of charge and structure, mostly the electronic aspect of this interplay has been investigated so far. However, to gain a fundamental understanding of this interplay, it is necessary to also elucidate the geometric aspects: for example, how does an excess charge couple to the various phonon modes of the system? In particular, to gain predictive power, e.g., to engineer hopping conduction, it is necessary to understand how the molecular structure reacts to additional charges. The functionalization of the tip of an AFM with a CO molecule allows imaging of the chemical structure of single molecules [27]. This opened the door for the characterization of adsorption configurations [28], bond-order relations, and geometrical changes upon self-assembly and chemical reactions with subangstrom resolution [29-34]. Here, we exploited AFM with CO-functionalized tips, in combination with $a b$ initio calculations, to study and quantify minute structural changes upon electron injection into an individual copper(II)phthalocyanine (CuPc) molecule adsorbed on ultrathin $\mathrm{NaCl}$. Subtle variations in the AFM contrast between the two charge states are visualized through difference images at various tip-sample distances. A comparison with simulated AFM images reveals that the contrast differences originate predominantly from a nonhomogeneous vertical relaxation of the molecule upon charging. This paves the way to the subangstrom determination of intramolecular structural relaxations occurring in molecular systems upon electron transfer. The experiments were performed with a home-built combined STM and qPlus-based AFM [35-37] operating at a base temperature of $7 \mathrm{~K}$ under ultrahigh vacuum $\left(p \approx 2 \times 10^{-10} \mathrm{mbar}\right)$. Bias voltages refer to the sample with respect to the tip. The AFM was operated in frequency-modulation mode [38], where the tip-sample interaction results in a frequency change $\Delta f$ of the qPlus sensor (quality factor $Q \approx 20000$; stiffness $k_{\mathrm{qPlus}} \approx 1.8 \mathrm{kN} / \mathrm{m}$; resonance frequency $f_{\text {res }} \approx$ $29 \mathrm{kHz})$. The oscillation amplitude was $0.5 \AA$. A $\mathrm{Cu}(100)$ surface as a substrate was prepared by sputtering $\left(\mathrm{Ne}^{+}, 1 \mathrm{keV}\right)$ and annealing cycles $(820 \mathrm{~K})$ followed by deposition of $\mathrm{NaCl}$. $\mathrm{CuPc}$ molecules were sublimed onto $\mathrm{NaCl} / \mathrm{Cu}(100)$ at $7 \mathrm{~K}$ and $\mathrm{CO}$ molecules at very low 
coverage were co-adsorbed for tip termination [27]. All AFM images were acquired at constant height (i.e., with a disabled feedback loop) with a CO-functionalized tip at zero bias for molecules adsorbed on a two monolayer (2 ML) $\mathrm{NaCl}$ film. Whereas $\mathrm{CuPc}$ can adsorb both centered at $\mathrm{Cl}^{-}$and at $\mathrm{Na}^{+}$, charge bistability occurs only in the latter adsorption site $[13,16]$, which is therefore the only one considered here. In this configuration the four isoindole units are aligned with the nonpolar directions of $\mathrm{NaCl}$. The experimental tip heights are provided as $z$ offsets relative to the constant-current STM set point, where a positive value ( $z$ offset $>0$ ) indicates a displacement away from the sample. Density functional theory (DFT) calculations were performed using the FHI-aims code with numerical atomic orbitals as the basis functions [39]. The Heyd-ScuseriaErnzerhof (HSE) [40] exchange-correlation functional with an admixture of exact exchange of 0.8 and a van der Waals $(\mathrm{vdW})$ method $[41,42]$ was applied for all calculations. AFM image simulations are based on a $\mathrm{vdW}$ attraction derived from the semiempirical potential [43] and Pauli repulsion calculated from the electron densities from DFT [44]. The latter are converted to a frequency shift using the experimental $k_{\mathrm{qPlus}}$. Lateral distortions due to the CO-functionalized tip [45] are simulated using a lateral spring constant of $k_{\mathrm{CO}}=$ $0.14 \mathrm{~N} / \mathrm{m}$ [46]. Relative tip height changes in experiments are well controlled and were enforced in the simulations, only the global offset to an absolute tip height was adjusted in the simulations. Tip heights of the latter refer to distances between the $\mathrm{CuPc}$ molecular plane and the center of carbon in the CO tip. For better comparison, the attractive $\Delta f$ background value from the clean surface was subtracted from the AFM images shown in Figs. 2-4.

The charge bistability in CuPc [see Fig. 1(a) for model] arises from its electron affinity being roughly aligned with the Fermi level of the $\mathrm{NaCl} / \mathrm{Cu}(100)$ substrate $[13,16]$. $I(V)$ spectra acquired above a $\mathrm{CuPc}$ molecule exhibit hysteresis [see Fig. 1(b)]. In the forward voltage sweep the current drop around $200 \mathrm{mV}$ indicates that the molecule becomes negatively charged [13], whereas the discharging event in the reverse sweep direction occurs at a lower bias voltage $(\approx-100 \mathrm{mV})$. Within the hysteresis loop, which arises from the reorganization energy [25], the molecule is bistable and can be deliberately imaged in both of the charge states. Figure 1(c) shows one of the two degenerate lowest unoccupied molecular orbitals (LUMO) as calculated for the neutral molecule in the gas phase. Figures 1(d) and 1(e) show zero-bias constant-height CO-tip AFM images of two CuPc molecules, where only one of them was selectively charged between the image acquisitions. To highlight the changes in the AFM contrast upon charging, the second image was subtracted from the first. The difference is shown in Fig. 1(f), exhibiting distinct intramolecular contrast for the molecule that became charged.
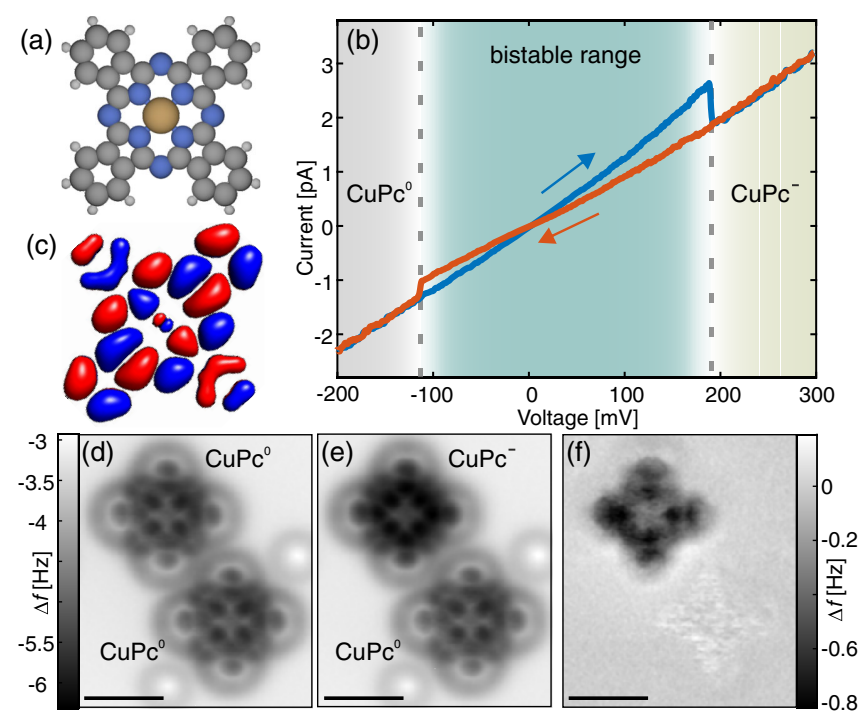

FIG. 1. (a) Model of a CuPc molecule. Hydrogens (white), carbons (gray), nitrogens (blue), and copper (brown). (b) $I(V)$ spectra of a CuPc molecule on $\mathrm{NaCl}(2 \mathrm{ML}) / \mathrm{Cu}(100)$. (c) LUMO of $\mathrm{CuPc}$. Constant-height AFM images recorded with a CO tip of (d) two neutral CuPc and (e) after charging the top-left molecule with a single electron. (f) Difference image [(d)-(e)]. AFM images were recorded with a $z$ offset of $1.65 \AA$ with respect to the STM set point $(I=1 \mathrm{pA}, V=-0.2 \mathrm{~V})$. Scale bars correspond to $10 \AA$.

This change in intramolecular contrast can therefore be ascribed to the addition of a single electron. Figure 2 shows such difference imaging at even higher resolution, where red and yellow crosses mark the positions of the bond ridges corresponding to the peripheral rings. Importantly, all markers are at identical positions in all three images, such that contrast differences in Fig. 2(c) can be attributed to different regions in Figs. 2(a) and 2(b). A comparison shows that the contrast in Figs. 2(a) and 2(b) varies in intensity, but does not shift laterally. Hence, image distortions due to $\mathrm{CO}$ bending [46-52] at the tip apex are similar in both cases and the difference image in Fig. 2(c) is not dominated by $\mathrm{CO}$ bending. If the contrast differences were dominated instead by image distortions, they should exhibit features that locally match a lateral derivative of the original images. The contrast in the difference image is incompatible with that, further indicating that $\mathrm{CO}$ bending is not dominant here. This is not surprising as the additional charge is rather delocalized over the entire molecule [13].

Beside other possible contributions, the charging will change the electrostatic contributions to the force [53-59] and will affect the local contact potential difference (LCPD), which can be quantified by Kelvin probe force spectroscopy (KPFS) [18,21,23]. However, the narrow voltage window of charge bistability inhibits the independent extraction of LCPD from KPFS for the two different charge states. Irrespective of the above, due to the fourfold symmetry of the difference image, the latter cannot be 

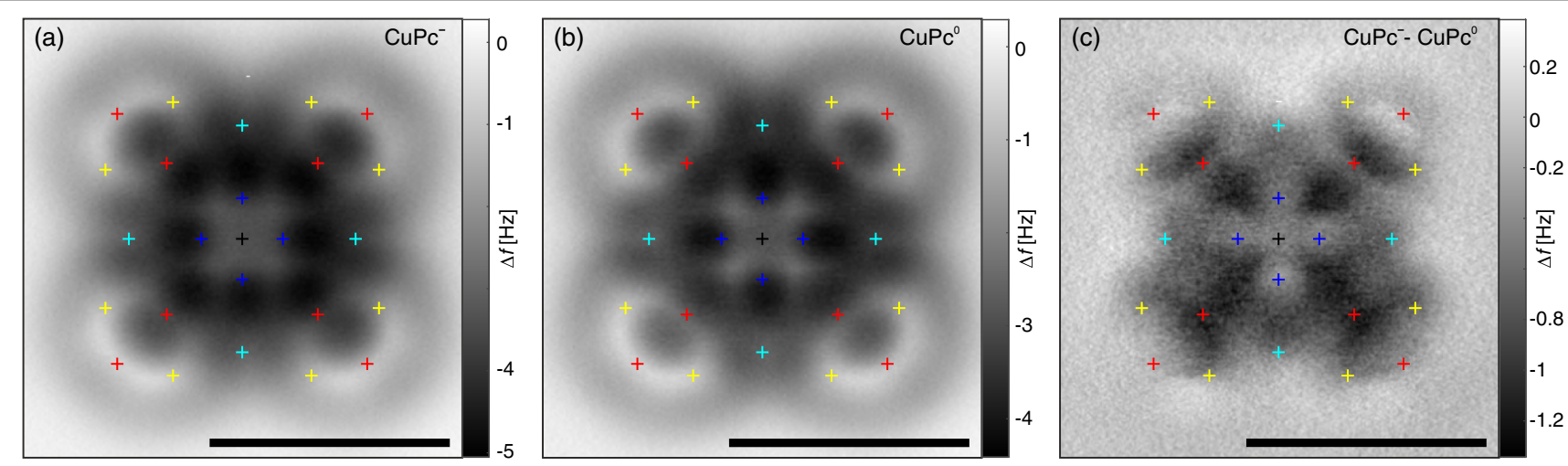

FIG. 2. High-resolution AFM images recorded with a $\mathrm{CO}$ tip of (a) $\mathrm{CuPc}^{-}$and (b) $\mathrm{CuPc}^{0}$. Both images were recorded at the same tip height $(z$ offset $=1.6 \AA$ with respect to the STM set point of $1.1 \mathrm{pA}$ at $90 \mathrm{mV})$. (c) Difference image $\left(\mathrm{CuPc}^{-}-\mathrm{CuPc}^{0}\right)$. Colored crosses indicate identical positions in all the AFM images to facilitate the comparison. Scale bars correspond to $10 \AA$.

simply ascribed to the charge distribution of the additional electron, since the occupied former LUMO of $\mathrm{CuPc}^{-}$is twofold symmetric [13] [see Fig. 1(c)]. Instead, the fourfold symmetric contrast difference shows mainly two distinct features: except at the center, the contrast difference shows some resemblance to the inverse of the intramolecular contrast of the original AFM images. This hints at a large influence of a vertical relaxation of the molecule upon charging: if the charged molecule was adsorbed closer to the surface than the neutral one (see also below), the shortranged Pauli repulsion between tip and molecule would be decreased such that the intramolecular contrast is weakened. The difference image is then expected to show an inverted intramolecular contrast. This seems to qualitatively explain the contrast in the difference image except for the molecule's center. To shed more light onto the contrast change upon charging, we performed DFT calculations to determine the geometries of a $\mathrm{CuPc}$ molecule adsorbed on $\mathrm{NaCl}(100)$ in both charge states. AFM images are simulated [44] based on the DFT-calculated geometries, and difference images were calculated analogous to the experiment. We compare experiment and simulation in different imaging regimes, by acquiring a set of constant-height AFM images of the same molecule in both charge states similar to the ones shown in Fig. 2 but at various different tip heights. Figure 3 shows this comparison for three imaging regimes ( $z$ offset $=1.6,2.1$, and $2.6 \AA$ ). For each of the distances, the experimental AFM images of $\mathrm{CuPc}^{0}$ [Figs. 3(a)-3(c)], the corresponding experimental [Figs. 3(d)-3(f)], and simulated [Fig. 3(g)-3(i)] difference images are displayed. The images of $\mathrm{CuPc}^{0}$ show that at close distance [Fig. 3(c)] the contrast consists of an attractive smooth background from vdW interactions superimposed with repulsive contributions from Pauli repulsion exhibiting intramolecular features. Because of the short-range nature of Pauli repulsion, at comparably large distances [Fig. 3(a)] only the vdW background prevails. Remarkably, the key features are well reproduced by the simulations at all tip heights. In the attractive regime [Figs. 3(d) and 3(g)], both experiment and simulation show a bright feature enclosed in the cyan markers. The experimental difference image displays faint depressions at the periphery, which are barely visible in the simulations. In the intermediate regime [Figs. 3(e) and 3(h)], experiment and simulations consistently show a bright feature enclosed in red and yellow markers and a bright cross in the center (black and blue markers). At close distances and increased intramolecular contrast, similar observations can be made
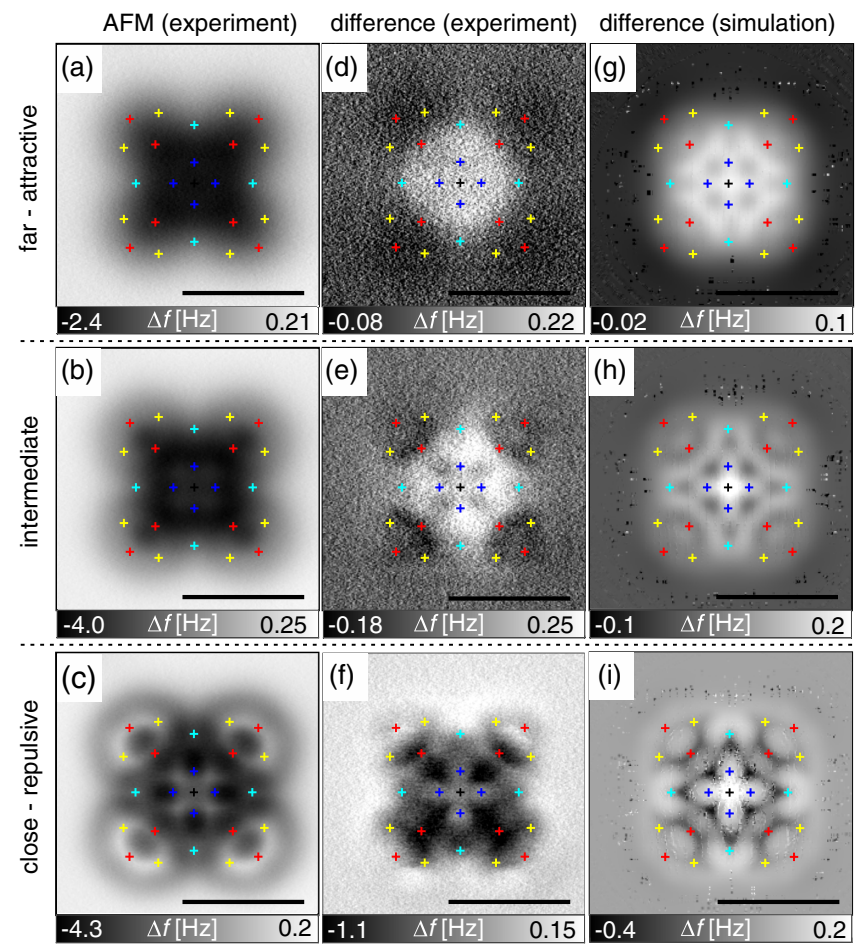

FIG. 3. (a)-(c) Constant-height AFM images of $\mathrm{CuPc}^{0}$ at different tip heights. (d)-(f) $\mathrm{CuPc}^{-}-\mathrm{CuPc}^{0}$ difference images from experimental AFM data and (g)-(i) from simulated AFM images. $z$ offsets are (a),(d) 2.6, (b),(e) 2.1, and (c),(f) $1.6 \AA$ with respect to a STM set point of $1.1 \mathrm{pA}$ and $90 \mathrm{mV}$. Tip heights for simulated images are (g) 5.8, (h) 5.3, and (i) $4.8 \AA$. Scale bars correspond to $10 \AA$. 


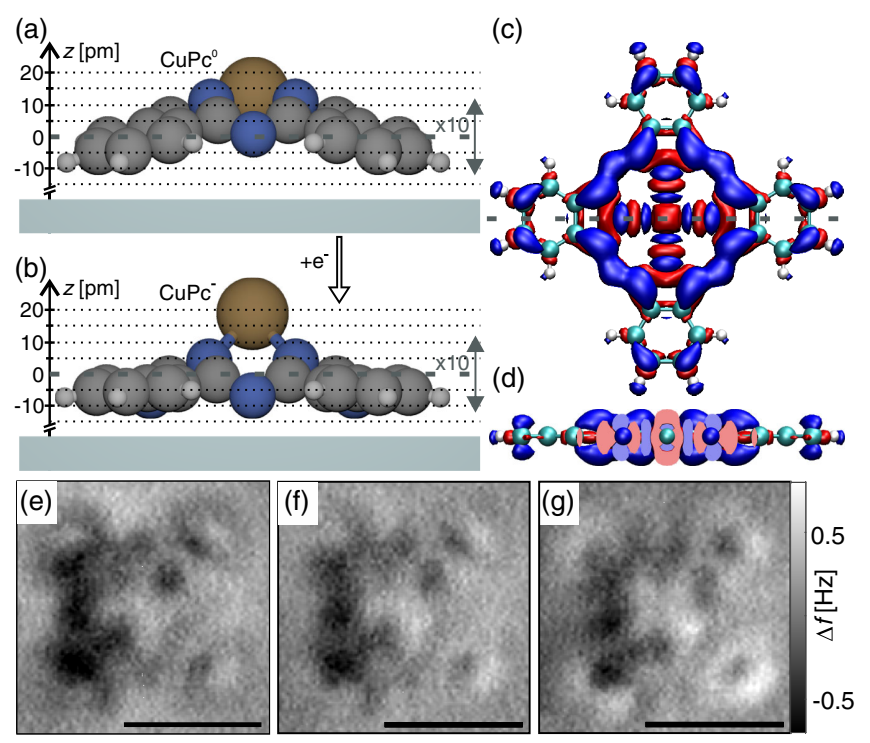

FIG. 4. 3D models showing the geometry of (a) $\mathrm{CuPc}^{0}$ and (b) $\mathrm{CuPc}^{-}$. The differences of the vertical coordinates of both species with respect to a reference plane (dashed line) are displayed magnified by a factor of 10 along the vertical direction. Charge density difference maps of a free $\mathrm{CuPc}$ molecule (c) top view and (d) cut through the molecule. Blue and red corresponds to an increase and decrease of electron density, respectively. The dashed line in (c) indicates the position of the cut through the molecule. The backbone of the molecule is shown for clarity. (e)-(g) difference images of CuPc with $\Delta z=-0.05$ (e), -0.10 (f), and $-0.15 \AA$ (g).

[Figs. 3(f) and 3(i)]. Generally, most features look more blurred in the experimental data, which we attribute to the finite size of the tip, which is not accounted for in the simulations. Likewise, electrostatic interactions between tip and molecule are not included in the simulations. As the charge is delocalized and electrostatic forces are comparatively long range in nature, we expect such electrostatic contributions to the AFM signal to be relatively homogeneous over the molecule. In fact, the remaining differences between experiment and simulations are in line with such a homogeneous background. We note that the very small oscillation amplitude of $0.5 \AA$ used, renders the measurements more sensitive to short-range Pauli repulsion in comparison to the long-range electrostatic contributions [37]. Moreover, in probe-particle image simulations $[51,60] \mathrm{CO}$-functionalized tips were approximated with a zero monopole charge for best agreement with experiment, suggesting that $\mathrm{CO}$-functionalized tips are comparably insensitive to electrostatics. A more detailed analysis of the electrostatic contributions to the image contrast can be found in the Supplemental Material [61]. The calculated geometries of the molecule in both charge states are analyzed in Fig. 4. As the relaxations and also the deviations from a planar geometry are tiny, for plotting we magnified them by a factor of 10 with respect to a plane of average height of atoms in $\mathrm{CuPc}^{0}$. In the neutral state
[Fig. 4(a)], the molecule has an umbrellalike geometry, being bent towards the $\mathrm{NaCl}$ surface at the periphery. Upon charging [Fig. 4(b)] the molecule relaxes towards the substrate and flattens out, only the $\mathrm{Cu}$ atom relaxes by $5 \mathrm{pm}$ away from the surface. The height of the outmost carbons and hydrogens almost remains unchanged. This relaxation pattern rationalizes the observed contrast change in the images in Fig. 2. Whereas the very periphery looks almost identical in the AFM images, the contrast inversion in the difference image is in line with the vertical relaxation towards the surface as mentioned further above. The opposite relaxation of the central $\mathrm{Cu}$ atom is also in agreement with the experimental observations of an increased $\Delta f$ at the very center. The contrast change in the other distance regimes can be rationalized along similar lines. We note that the contrast change at the central metal atoms-although qualitatively reproduced-seems overestimated in the simulations.

The observed relaxation pattern can be interpreted in light of the difference in charge densities between $\mathrm{CuPc}^{-}$and $\mathrm{CuPc}^{0}$ as calculated from DFT and shown in Figs. 4(c)-4(d). The charge density is increased in the $\pi$ system close to the molecule's center around the $\mathrm{Cu}$ atom. At the $\mathrm{Cu}$ atom, in contrast, the charge density appears to be decreased. Since the $\mathrm{Cu}$ is situated above a $\mathrm{Na}^{+}$ion, the decreased electron density causes a local repulsive electrostatic contribution that pushes the $\mathrm{Cu}$ atom upwards. The strongest increase in charge density occurs at the central unit of $\mathrm{CuPc}$, whereas at the periphery the charge density difference is much smaller. In general, the interaction of neutral molecules with insulating films comes mainly from $\mathrm{vdW}$ forces. Electrostatic forces can-in the case of polar functional groups or local charges-also play a significant role for the adsorption energy of molecules [62-67]. Further stabilization can arise from screening in the substrate. The extra electron in our case gives rise to such an additional electrostatic contribution and the nonhomogeneous distribution of the additional charge in $\mathrm{CuPc}^{-}$ agrees well with the relaxations described above. In STM experiments we observe a twofold symmetric appearance of the lowest-lying electronic resonance when tunneling into the already charged $\mathrm{CuPc}^{-}$, in agreement with previous STM experiments [13], indicating that the extra electron populates one of the former LUMOs of CuPc. The DFT calculations, in contrast, claim that the excess charge in $\mathrm{CuPc}^{-}$populates the former singly occupied $b_{1 g}$ orbital being more strongly localized at the center. This may explain why the contrast change at the very center is overestimated in the simulations. We note that the question, which orbital the excess electron occupies is governed by a subtle energy balance, in which the Coulombic charging energy depends on the degree of delocalization [68], the surrounding [16], and screening. It is known that different DFT functionals favor delocalization to different degree. We note that DFT calculations for the free anion in the gas 
phase provide a different population of states as compared to the adsorbed case. Finally, we demonstrate how the vertical relaxation of $\mathrm{CuPc}$ upon charging can be estimated from experimental data alone. To this end, we subtract AFM images of $\mathrm{CuPc}^{0}$ acquired at different $z$ offsets from $\mathrm{CuPc}^{-}$at a fixed $z$ offset. The difference in $z$ offset between the subtracted images we define as $\Delta z$, where $\Delta z<0$ corresponds to a smaller tip height for $\mathrm{CuPc}^{-}$. The concept of this analysis is that if the vertical relaxation of the molecule is compensated by a $\Delta z$, the intramolecular contrast arising from Pauli repulsion should be similar in both images and thus cancel out when taking their difference. Figures 4(e)-4(g) show such difference images, for three different $\Delta z$. The contrast inverses from $\Delta z=-0.05$ to $-0.15 \AA$ and cancels out best at $\Delta z \approx-0.1 \AA$, suggesting an average relaxation on the order of $-0.1 \AA$ upon charging. This value nicely fits the vertical relaxations predicted by theory. The remaining contrast in the images is in line with a nonhomogenous relaxation of the molecule. The fact that the vertical relaxations are stronger at the center than in the periphery of the molecule can qualitatively already be deduced directly from the difference image shown in Fig. 2. This analysis shows that vertical relaxation can be extracted with a precision of about $5 \mathrm{pm}$ based on experimental AFM data. In summary, we demonstrate how charging individual CuPc molecules by a single electron impacts submolecularly resolved AFM images. The difference in contrast for the two charge states (neutral vs anionic) appears to be only very weakly influenced by the charge density difference directly, but rather dominated by vertical relaxations of the molecule. These relaxations-although tiny-lead to appreciable contrast changes well above the experimental noise floor, highlighting the large sensitivity of AFM to relaxations at the pm scale. DFT calculations confirm that the relaxation pattern is not uniform over the molecule. Our results demonstrate the possibility to determine subangstrom geometric changes in molecular systems upon electron transfer.

We thank Florian Albrecht and Ferdinand Evers for discussion. Financial support from the Deutsche Forschungsgemeinschaft (No. RE2669/6-1, GRK 1570), Marie Curie Initial Training Network MOLESCO (No. 606728), and European Research Council Consolidator Grant AMSEL (Agreement No. 682144) are gratefully acknowledged.

*Philipp.Scheuerer@ur.de †NIM@zurich.ibm.com

[1] N. S. Sariciftci, L. Smilowitz, A. J. Heeger, and F. Wudl, Science 258, 1474 (1992).

[2] R. A. Marcus, Rev. Mod. Phys. 65, 599 (1993).

[3] D. I. Gittins, D. Bethell, D. J. Schiffrin, and R. J. Nichols, Nature (London) 408, 67 (2000).
[4] W. Haiss, H. van Zalinge, S. J. Higgins, D. Bethell, H. Höbenreich, D. J. Schiffrin, and R. J. Nichols, J. Am. Chem. Soc. 125, 15294 (2003).

[5] F. Chen, J. He, C. Nuckolls, T. Roberts, J. E. Klare, and S. Lindsay, Nano Lett. 5, 503 (2005).

[6] G. R. Hutchison, M. A. Ratner, and T. J. Marks, J. Am. Chem. Soc. 127, 2339 (2005).

[7] K. Susumu, P. R. Frail, P. J. Angiolillo, and M. J. Therien, J. Am. Chem. Soc. 128, 8380 (2006).

[8] S. Ho Choi, B. Kim, and C. D. Frisbie, Science 320, 1482 (2008).

[9] M. Sterrer, T. Risse, U. Martinez, L. Giordano, M. Heyde, H.-P. Rust, G. Pacchioni, and H.-J. Freund, Phys. Rev. Lett. 98, 096107 (2007).

[10] Y.-S. Fu, T. Zhang, S.-H. Ji, X. Chen, X.-C. Ma, J.-F. Jia, and Q.-K. Xue, Phys. Rev. Lett. 103, 257202 (2009).

[11] J. Park, B. D. Yu, and H. Kim, Phys. Rev. B 79, 233407 (2009).

[12] S. J. van der Molen and P. Liljeroth, J. Phys. Condens. Matter 22, 133001 (2010).

[13] I. Swart, T. Sonnleitner, and J. Repp, Nano Lett. 11, 1580 (2011).

[14] T. Leoni, O. Guillermet, H. Walch, V. Langlais, A. Scheuermann, J. Bonvoisin, and S. Gauthier, Phys. Rev. Lett. 106, 216103 (2011).

[15] B. Huang, H. Xiang, J. Yu, and S.-H. Wei, Phys. Rev. Lett. 108, 206802 (2012).

[16] C. Uhlmann, I. Swart, and J. Repp, Nano Lett. 13, 777 (2013).

[17] J. Martínez-Blanco, C. Nacci, S. C. Erwin, K. Kanisawa, E. Locane, M. Thomas, F. von Oppen, P. W. Brouwer, and S. Fölsch, Nat. Phys. 11, 640 (2015).

[18] W. Steurer, J. Repp, L. Gross, I. Scivetti, M. Persson, and G. Meyer, Phys. Rev. Lett. 114, 036801 (2015).

[19] J. Repp, G. Meyer, F. E. Olsson, and M. Persson, Science 305, 493 (2004).

[20] F. E. Olsson, S. Paavilainen, M. Persson, J. Repp, and G. Meyer, Phys. Rev. Lett. 98, 176803 (2007).

[21] L. Gross, F. Mohn, P. Liljeroth, J. Repp, F. J. Giessibl, and G. Meyer, Science 324, 1428 (2009).

[22] J. Repp, W. Steurer, I. Scivetti, M. Persson, L. Gross, and G. Meyer, Phys. Rev. Lett. 117, 146102 (2016).

[23] W. Steurer, S. Fatayer, L. Gross, and G. Meyer, Nat. Commun. 6, 8353 (2015).

[24] A. Kumar, K. Banerjee, M. Dvorak, F. Schulz, A. Harju, P. Rinke, and P. Liljeroth, ACS Nano 11, 4960 (2017).

[25] S. Fatayer, B. Schuler, W. Steurer, I. Scivetti, J. Repp, L. Gross, M. Persson, and G. Meyer, Nat. Nanotechnol. 13, 376 (2018).

[26] L. L. Patera, F. Queck, P. Scheuerer, and J. Repp, Nature (London) 566, 245 (2019).

[27] L. Gross, F. Mohn, N. Moll, P. Liljeroth, and G. Meyer, Science 325, 1110 (2009).

[28] B. Schuler, W. Liu, A. Tkatchenko, N. Moll, G. Meyer, A. Mistry, D. Fox, and L. Gross, Phys. Rev. Lett. 111, 106103 (2013)

[29] L. Gross, F. Mohn, N. Moll, B. Schuler, A. Criado, E. Guitián, D. Peña, A. Gourdon, and G. Meyer, Science 337, 1326 (2012). 
[30] N. Pavliček, B. Fleury, M. Neu, J. Niedenführ, C. HerranzLancho, M. Ruben, and J. Repp, Phys. Rev. Lett. 108, 086101 (2012).

[31] D. G. de Oteyza, P. Gorman, Y.-C. Chen, S. Wickenburg, A. Riss, D. J. Mowbray, G. Etkin, Z. Pedramrazi, H.-Z. Tsai, A. Rubio, M. F. Crommie, and F. R. Fischer, Science 340, 1434 (2013).

[32] F. Albrecht, N. Pavliček, C. Herranz-Lancho, M. Ruben, and J. Repp, J. Am. Chem. Soc. 137, 7424 (2015).

[33] L. L. Patera, X. Liu, N. Mosso, S. Decurtins, S.-X. Liu, and J. Repp, Angew. Chem., Int. Ed. 56, 10786 (2017).

[34] F. Queck, O. Krejčí, P. Scheuerer, F. Bolland, M. Otyepka, P. Jelínek, and J. Repp, J. Am. Chem. Soc. 140, 12884 (2018).

[35] F. J. Giessibl, Phys. Rev. B 56, 16010 (1997).

[36] F. J. Giessibl, Appl. Phys. Lett. 73, 3956 (1998).

[37] F. J. Giessibl, Appl. Phys. Lett. 76, 1470 (2000).

[38] T. R. Albrecht, P. Grütter, D. Horne, and D. Rugar, J. Appl. Phys. 69, 668 (1991).

[39] V. Blum, R. Gehrke, F. Hanke, P. Havu, V. Havu, X. Ren, K. Reuter, and M. Scheffler, Comput. Phys. Commun. 180, 2175 (2009).

[40] J. Heyd, G. E. Scuseria, and M. Ernzerhof, J. Chem. Phys. 118, 8207 (2003).

[41] A. Tkatchenko and M. Scheffler, Phys. Rev. Lett. 102, 073005 (2009).

[42] V. G. Ruiz, W. Liu, E. Zojer, M. Scheffler, and A. Tkatchenko, Phys. Rev. Lett. 108, 146103 (2012).

[43] S. Grimme, J. Comput. Chem. 27, 1787 (2006).

[44] N. Moll, L. Gross, F. Mohn, A. Curioni, and G. Meyer, New J. Phys. 14, 083023 (2012).

[45] C.-S. Guo, M. A. Van Hove, X. Ren, and Y. Zhao, J. Phys. Chem. C 119, 1483 (2015).

[46] M. Neu, N. Moll, L. Gross, G. Meyer, F. J. Giessibl, and J. Repp, Phys. Rev. B 89, 205407 (2014).

[47] N. Moll, L. Gross, F. Mohn, A. Curioni, and G. Meyer, New J. Phys. 12, 125020 (2010).

[48] N. Moll, B. Schuler, S. Kawai, F. Xu, L. Peng, A. Orita, J. Otera, A. Curioni, M. Neu, J. Repp, G. Meyer, and L. Gross, Nano Lett. 14, 6127 (2014).

[49] P. Hapala, G. Kichin, C. Wagner, F. S. Tautz, R. Temirov, and P. Jelínek, Phys. Rev. B 90, 085421 (2014).

[50] M. P. Boneschanscher, S. K. Hämäläinen, P. Liljeroth, and I. Swart, ACS Nano 8, 3006 (2014).
[51] P. Hapala, M. Švec, O. Stetsovych, N. J. van der Heijden, M. Ondráček, J. van der Lit, P. Mutombo, I. Swart, and P. Jelínek, Nat. Commun. 7, 11560 (2016).

[52] J. van der Lit, F. Di Cicco, P. Hapala, P. Jelinek, and I. Swart, Phys. Rev. Lett. 116, 096102 (2016).

[53] C. Schönenberger and S. F. Alvarado, Phys. Rev. Lett. 65, 3162 (1990).

[54] L. J. Klein and C. C. Williams, Appl. Phys. Lett. 79, 1828 (2001).

[55] M. T. Woodside and P. L. McEuen, Science 296, 1098 (2002).

[56] R. Stomp, Y. Miyahara, S. Schaer, Q. Sun, H. Guo, P. Grutter, S. Studenikin, P. Poole, and A. Sachrajda, Phys. Rev. Lett. 94, 056802 (2005).

[57] L. Cockins, Y. Miyahara, S. D. Bennett, A. A. Clerk, and P. Grutter, Nano Lett. 12, 709 (2012).

[58] P. Rahe, R. P. Steele, and C. C. Williams, Nano Lett. 16, 911 (2016).

[59] M. Rashidi, W. Vine, T. Dienel, L. Livadaru, J. Retallick, T. Huff, K. Walus, and R. A. Wolkow, Phys. Rev. Lett. 121, 166801 (2018).

[60] O. Krejčí, P. Hapala, M. Ondráček, and P. Jelínek, Phys. Rev. B 95, 045407 (2017).

[61] See Supplemental Material at http://link.aps.org/ supplemental/10.1103/PhysRevLett.123.066001 for an analysis of the electrostatic contributions to the image contrast.

[62] T. Trevethan and A. L. Shluger, J. Phys. Chem. C 112, 19577 (2008).

[63] C. Barth, M. Gingras, A. S. Foster, A. Gulans, G. Félix, T. Hynninen, R. Peresutti, and C. R. Henry, Adv. Mater. 24, 3228 (2012).

[64] P. Rahe, M. Nimmrich, and A. Kühnle, Small 8, 2969 (2012).

[65] C. M. Hauke, R. Bechstein, M. Kittelmann, C. Storz, A. F. M. Kilbinger, P. Rahe, and A. Kühnle, ACS Nano 7, 5491 (2013).

[66] P. Rahe, M. Kittelmann, J. L. Neff, M. Nimmrich, M. Reichling, P. Maass, and A. Kühnle, Adv. Mater. 25, 3948 (2013).

[67] B. Hoff, M. Gingras, R. Peresutti, C. R. Henry, A. S. Foster, and C. Barth, J. Phys. Chem. C 118, 14569 (2014).

[68] M.-S. Liao and S. Scheiner, J. Chem. Phys. 114, 9780 (2001). 